\section{Management of the patient with sepsis in emergency department: a new alternative protocol}

\author{
Manuel Monti, ${ }^{1}$ Lucia Stefanecchia, ${ }^{1}$ \\ Igino Fusco Moffa, ${ }^{2}$ Luciano Fioriti, ${ }^{3}$ \\ Manolo Filippucci, ${ }^{1}$ \\ Giovanni Maria Vincentelli, ${ }^{4}$ \\ Francesco Borgognoni ${ }^{1}$ \\ 1Emergency Department, Local Health \\ Unit Umbria 1, Assisi; ${ }^{2}$ Prevention \\ Department, Local Health Unit Umbria 1, \\ Assisi; ${ }^{3}$ Medical Laboratory, Local Health \\ Unit Umbria 1, Assisi; ${ }^{4}$ Emergency \\ Department, San Giovanni Calibita - \\ Fatebenefratelli Hospital, Isola Tiberina, \\ Rome, Italy
}

\section{Abstract}

Sepsis is a clinical syndrome induced from the host response to an infection. Severe sepsis is the leading cause of death in critically ill patients. The introduction of the early goaldirected therapy (EGDT) has been able to reduce mortality in patients with severe sepsis/septic shock. However, sepsis mortality rates remain high compared to other critical illnesses. Many studies have pointed out that the use of arterial line placement and the execution of central venous pressure and central venous oxygen saturation measurements are the most difficult EGDT elements to carry out in community hospitals. For these reasons, the present independent review examines recent pathogenic, diagnostic, and therapeutic development in sepsis with particular relevance to the emergency practice, following the latest guidelines published in February 2013 and several recent studies. We propose a non-invasive alternative protocol which can replace the standard treatment with non-substantial changes in the patient outcome though overcoming the obstacles of a invasive method.

\section{Introduction}

The progression of sepsis towards an unfavorable outcome depends mainly on delayed diagnosis and treatment, on the virulence of the pathogen and on the immune status of the patient. ${ }^{1}$ Jones has recently defined sepsis as a complex puzzle, where the host response to the infection is characterized by different reactions such as inflammation, endothelial dysfunction, alterations to the coagulation-fibri- nolysis system and others. ${ }^{2}$ The course of the disease can lead to severe sepsis, multiple organs failure, hypotension, and septic shock. ${ }^{3}$

The hospitalization rate of people with a principal diagnosis of septicemia or sepsis more than doubled from 2000 through 2008, increasing from 11.6 to 24.0 per 10,000 people with a prevalence of gram-negative infections. ${ }^{4}$ Patterns of infecting organisms were similar to those in previous studies, with predominant organisms being Staphylococcus aureus (20.5\%), Pseudomonas species (19.9\%), Enterobacteriacae (mainly E. coli, 16.0\%), and fungi $(19 \%) .^{5}$

The early goal-directed therapy (EGDT) is often underused partly due to the difficulty in invasive procedures. The use of a non-invasive protocol to facilitate the implementation of EGDT may be an effective strategy to improve protocol adherence. ${ }^{6}$ The identification of septic patients during the golden hour is very important, so the EGDT treatment is correlated to a favorable outcome (Figure 1). ${ }^{7}$

\section{Diagnosis}

During the triage it is important to identify early signs of infections and hypotension/hypoperfusion through an accurate medical history and physical examination, using the standard ABCD protocol. At the end of the evaluation, a priority colour can be assigned to the patient (Figure 2).

The first management steps for a patient attending the accident \& emergency (A\&E) department or hospitalized with a suspected sepsis are:1-8 i) recognition of the systemic inflammatory response syndrome (SIRS); identification of a single infective episode sure or probable (sepsis); evaluation of organs failure (severe sepsis) or severe hypoperfusion (septic shock) (Figure 3).

During the first medical assessment, it is necessary to perform: ${ }^{9}$ i) recording of a detailed medical history and complete physical examination; ii) detection of vital functions (blood pressure, heart rate, respiratory frequency, $\mathrm{SpO}_{2}$, body temperature); iii) blood tests: full blood count, kidney liver pancreas functional tests, coagulation, inflammatory markers, blood gas analysis including anion gap and lactate dosage, hemocultures if needed; iv) electrocardiogram; v) chest X-ray, abdomen ultrasound scan, rachicentesis and computed tomography scan of the head if clinically required); vi) start treatment, if required.

The performance of blood cultures from 2 peripheral venous access for the identification of aerobic/anaerobic bacteria is very important (if such cultures do not cause a significant delay $>45 \mathrm{~min}$ ) and it is absolutely advisable to
Correspondence: Manuel Monti, Emergency Department, Local Health Unit Umbria 1,

Via V. Muller 1, Assisi (PG), Italy.

Tel. +39.334.6617176 - Fax: +39.075.5412205.

E-mail: montimanuel@tiscali.it

Key words: septic shock, non-invasive treatment, EGDT, clearance lactate.

Received for publication: 17 January 2014.

Accepted for publication: 7 May 2014.

This work is licensed under a Creative Commons Attribution 3.0 License (by-nc 3.0).

(C) Copyright M. Monti et al., 2014

Licensee PAGEPress, Italy

Emergency Care Journal 2014; 10:2241

doi:10.4081/ecj.2014.2241

perform in the A\&E, although we must consider some technical difficulties. ${ }^{1}$ In patients with central venous access it is required to perform blood cultures of central and peripheral veins simultaneously, and reporting time and site of blood cultures. ${ }^{1}$ In presence of an urinary catheter or alterations of diuresis an urinalysis with urine culture must be performed. In presence of ulcers or wounds or evident cutaneous infection a skin swab for aerobic/anaerobic pathogens is required., ${ }^{1,8-10}$ Diagnostic procedures must be performed early in order to identify the source of infection and the pathogen responsible, with removal of infected devices, if necessary. ${ }^{11}$

\section{Treatment}

The initial treatment of a patient affected by sepsis has been described in the 2012 international guidelines, and it is based on the concept of bundle treatment. Bundle treatment refers to a series of therapeutic interventions administered for a disease, with a clear scientific evidence that they perform better in terms of outcome when compared to any of the single interventions. ${ }^{1}$ In the treatment of sepsis we can identify 2 types of bundle: ${ }^{1}$ i) the resuscitation bundle to be performed within $6 \mathrm{~h}$ and ii) the management bundle to be performed within $24 \mathrm{~h}$. The goal of the resuscitation bundle is the stabilization of the vital signs [mean arterial pressure (MAP), central venous pressure (CVP), oxygen that reaches the superior vena cava $\left(\mathrm{ScvO}_{2}\right)$, diuresis]. Several studies have complained about the lack of non-invasive methods to evaluate the efficacy of the resuscitation bundle. ${ }^{12-14}$

The pathogenesis of organ dysfunction is multifactorial and incompletely understood. Tissue hypoperfusion and hypoxia are domi- 
nant factors. ${ }^{15}$ In order to evaluate the hypoperfusion it is necessary to start from the physical examination (consciousness, temperature, jugular status, pulmonary oedema, peripheral oedema, vital signs, diuresis) followed by invasive (CVP monitor) or non-invasive tests. Several groups have observed that CVP alone fails to reliably predict a hemodynamic response to volume expansion and some patients with high CVP will respond to volume expansion. ${ }^{16-18}$ However, internationally endorsed clinical guidelines recommend using CVP as the end point of fluid resuscitation. In our opinion, considering the complexity of the physiologic feedback and clinical picture, robust reflexes and homeostatic mechanisms, CVP can be considered as a parameter for assessing the volume status. However, the hemodynamic status of the patient should be considered before deciding any therapeutic procedure. . $^{1,19,20}$

Invasive measurement of CVP has a number of limitations, including the fact that it is influenced by the ventricular compliance, intrathoracic pressure, contraction of central veins, resulting in an approximate estimate of the haemodynamic status of the patient. ${ }^{21}$ In addition, the use of invasive measurement needs experienced staff, which not always is present in the emergency department. Several authors have proposed the use of ultrasound to assess the diameter and the collapsibility of the inferior vena cava (IVC) as a parameter for the calculation of CVP. ${ }^{22,23}$ Its collapse index and CVP allow to classify a patient as empty when diameter of IVC is $\leq 15 \mathrm{~mm}$ and there is full collapse during inspiration corresponding to a $\mathrm{CVP} \leq 5$ mmHG. A full patient, on the other hand, reports a diameter of IVC $\geq 25 \mathrm{~mm}$, no collapse during inspiration corresponding to CVP 15-20 mmHG (Table 1). ${ }^{22,23}$ Normal values are consid- ered as a IVC diameter of $20 \mathrm{~mm}$ with an inspiratory collapse of $50 \%{ }^{24-26}$

We believe that in the emergency department it is absolutely appropriate to use the ultrasound technique as an alternative to invasive procedures, which are difficult to perform for logistical and technical problems. Emergency physicians found point-of-care ultrasonographic data about cardiac contractility, IVC diameter, and IVC collapsibility to be clinically useful in treating adult patients with sepsis. ${ }^{27}$ Another important issue to consider in a patient with sepsis is the tissue hypoxia. ${ }^{1}$ Hypoxia can be due to alterations to pre-load, post-load, myocardial contractility and $\mathrm{O}_{2}$ transport alterations. During hypoxia, cells in the tissues can extract more oxygen from the arterious blood, and this is particularly evident in the first phase of the sepsis. As a consequence, the amount of $\mathrm{ScvO}_{2}$ can be decreased (beginning of severe sepsis). ${ }^{24}$ When the alterations of the microcirculation are so severe that it is not anymore possible to extract an adequate level of oxygen from the blood, $\mathrm{ScvO}_{2}$ increases, marking the beginning of the septic shock. ${ }^{25}$ This means that, when cardiac output is low (and therefore it is a crucial clinical issue), cardiac output measurements are technically poor in reflecting tissue oxygenation. It is possible to measure the venous oxygen sat- uration through a catheter in inferior or superior vena cava with the exception of the coronary blood, resulting in an overestimation of the tissue oxygenation. It is however possible to monitor the myocardic hypoxia through the use of the Swan Graz in the pulmonary artery. ${ }^{26}$

A number of evidences has recently proved that it is possible to use the lactate clearance (a non-invasive method) instead of the $\mathrm{ScvO}_{2}$ to evaluate the efficacy of EGDT in A\&E departments. ${ }^{28-30}$ In 2010 Jones stated that the normalisation of the lactate clearance or of $\mathrm{ScvO}_{2}$ were comparable when monitoring their response to treatment, and that there was no difference between the two in terms of outcome when measuring the efficacy of the EGDT. ${ }^{31}$ The formula of the lactic acid clearance is the following:

Initial lactate - Subsequent lactate $2 \mathrm{~h}$ Initial lactate (\%)

A reduction of the clearance of the lactate $>10 \%$ in $2 \mathrm{~h}$ is considered to be comparable to achieving or maintaining a $\mathrm{ScvO}_{2}>70 \% .{ }^{32} \mathrm{We}$ recommend the use of lactate clearance as the most appropriate and convenient method to choose during the early assessment of the septic patient in the hospital when it is not practical to insert a venous catheter.

Table 1. Correlation between central venous pressure and inferior vena cava appearance.

\begin{tabular}{lccccc} 
& & \multicolumn{5}{c}{ CVP $(\mathrm{cm})$} \\
IVC on inspiration & Totally & Collapses & Collapses & Collapses & No \\
& collapses & $>50 \%$ & $<50 \%$ & $<50 \%$ & change \\
IVC diameter $(\mathrm{cm})$ & $<1.5$ & $1.5-2.5$ & $1.5-2.5$ & $>2.5$ & $>2.5$ \\
\hline
\end{tabular}

IVC, inferior vena cava; CVP, central venous pressure.

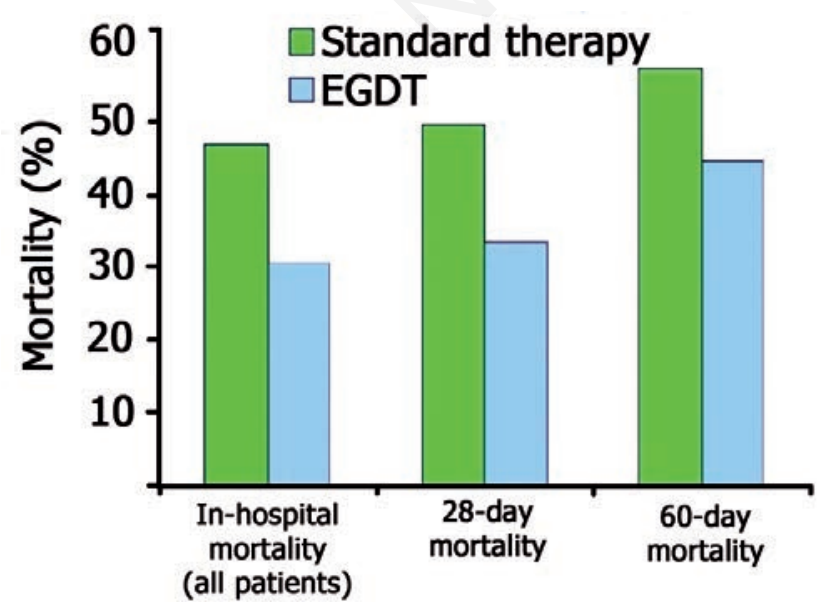

Figure 1. Efficacy of early goal-directed therapy in terms of mortality.

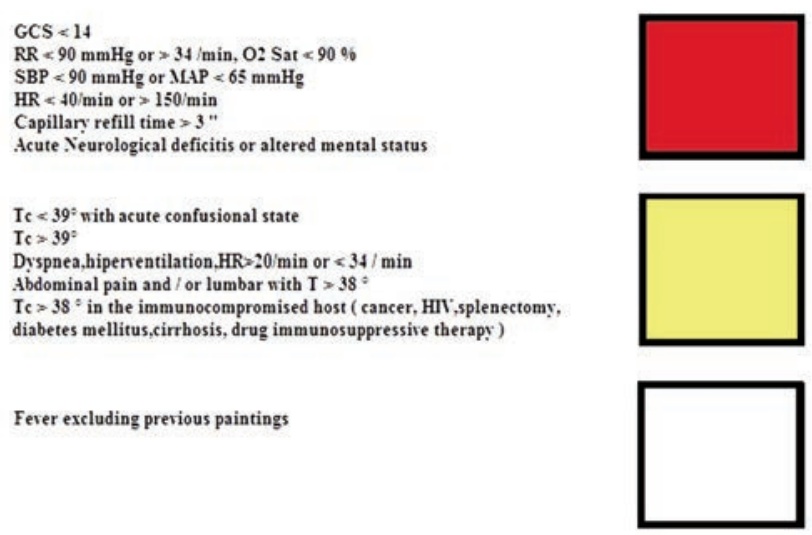

Figure 2. Triage protocol of the patient with suspected sepsis, based on our experience and on scientific literature. ${ }^{1,7}$ Parameter: GCS. Ap-HT. Temperature, RR, $\mathrm{O} 2$ sat. 
Patient exhibits two or more of the following SIRS criteria

Temperature $>38^{\circ} \mathrm{C}\left(100.4^{\circ} \mathrm{F}\right)$ or $<36^{\circ} \mathrm{C}\left(96.8^{\circ} \mathrm{F}\right)$

Heart rate $>90$ beats $/ \mathrm{min}$

Respiratory rate $\geq 20$ breaths/minute or $\mathrm{PaCO}_{2} \leq 32 \mathrm{mmHg}$

WBC $\geq 12,000 / \mathrm{mm}^{3}$ or $\leq 4000 / \mathrm{mm}^{3}$ AND suspected or proven infection

SIRS<smiles>C1CCCCC1</smiles>

SIRS with a presumed or confirmed infectious process

SEPSIS

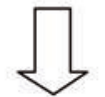

Sepsis with organ failure

SEVERE SEPSIS

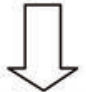

Refrectory hypotension

SEPTIC SHOCK

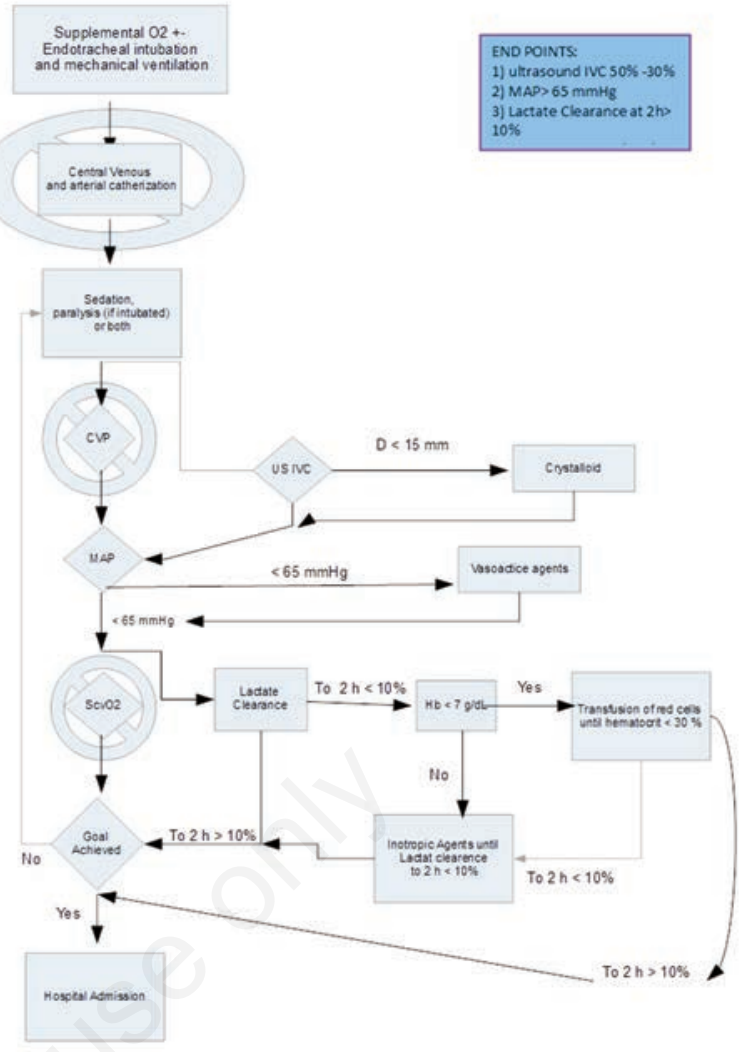

Figure 4. Our proposal for an alternative non invasive protocol.

Figure 3. Clinical and physiopathological classification of sepsis.

In conclusion, it is possible to develop a protocol of evaluation and treatment of the septic patient using non-invasive techniques: ultrasound (IVC, chest, heart) and clearance of the lactate (Figure 4).

\section{Conclusions}

The start of an early empiric treatment and of EGDT within $6 \mathrm{~h}$ is extremely important in patients with severe sepsis or septic shock. Monitoring the haemodynamic and perfusion status can now be achieved through the use of non-invasive techniques, very easy to use in A\&E.

\section{References}

1. Dellinger RP, Levy MM, Rhodes A, et al. Surviving sepsis campaign: international guidelines for management of severe sepsis and septic shock: 2012. Crit Care Med 2013;41:580-637.

2. Jones AE, Trzeciak S, Dellinger RP. Arterial pressure optimization in the treatment of septic shock: a complex puzzle. Crit Care
2010;14:102.

3. Levy MM, Fink MP, Marshall JC, et al. SCCM/ESICM/ACCP/ATS/SIS International sepsis definitions conference 2001. Crit Care Med 2003;29:530-8.

4. Mayr FB, Yende S, Angus DC. Epidemiology of severe sepsis. Virulence 2014;5:4-11.

5. Vincent JL, Rello J, Marshall J, et al. EPIC II group of investigators. International study of the prevalence and outcomes of infection in intensive care units. J Am Med Assoc 2009;302:2323.

6. De Miguel-Yanes JM, Andueza-Lillo JA, González-Ramallo VJ, et al. Failure to implement evidence-based clinical guidelines for sepsis at the ED. Am J Emerg Med 2006;24:553-9.

7. Rivers EP, Nguyen HB, Huang DT, Donnino M. Early goal directed therapy. Crit Care Med 2004;32:314-5.

8. Fauci AS, Morens DM. The perpetual challenge of infectious diseases. New Engl $\mathrm{J}$ Med 2012;366:454-61.

9. Levy MM, Fink MP, Marshall JC, et al. SCCM/ESICM/ACCP/ATS/SIS International sepsis definitions conference. Crit Care Med 2003;31:1250-6.

10. Chen Y-C, Jenq C-C, Tian Y-C, et al. Rifle classification for predicting in-hospital mortality in critically ill sepsis patients. Shock 2009;31:139-45.

11. Seam N, Suffredini AF. Mechanisms of sepsis and insights into clinical trials. Drug Discov Today 2007;4:83-93.

12. Rivers EP, Katranji M, Jaehne KA, et al. Early interventions in severe sepsis and septic shock: a review of the evidence one decade later. Minerva Anestesiol 2012;78:712-24.

13. Levinson AT, Casserly BP, Levy MM. Reducing mortality in severe sepsis and septic shock. Semin Resp Crit Care 2011;32:195-205.

14. Kruer RM, Ensor CR. Colloids in the intensive care unit. Am J Health-Syst Ph 2012;69:1635-42.

15. Cohen J. The immunopathogenesis of sepsis. Nature 2002;420:885-91.

16. Marik PE, Cavallazzi R. Does the central venous pressure predict fluid responsiveness? An updated meta-analysis and a plea for some common sense. Crit Care Med 2013;41:1774-81.

17. Shippy CR, Appel PL, Shoemaker WC. Reliability of clinical monitoring to assess blood volume in critically ill patients. Crit Care Med 1984;12:107-12.

18. Bendjelid K, Romand JA. Fluid responsiveness in mechanically ventilated patients: a 
review of indices used in intensive care. Intens Care Med 2003;29:352-60.

19. Magder S, Bafaqeeh F. The clinical role of central venous pressure measurements. Intens Care Med 2007;22:44-5.

20. Wiedemann HP, Wheeler AP, Bernard GR, et al. Comparison of two fluid-management strategies in acute lung injury. New Engl J Med 2006;354:2564-75.

21. Barochia AV, Cui X, Eichacker PQ. The surviving sepsis campaign's revised sepsis bundles. Curr Infect Dis 2013;15:385-93.

22. Vieillard-Baron A, Prin S, Chergui K, et al. Hemodynamic instability in sepsis: bedside assessment by Doppler echocardiography. Am J Resp Crit Care 2003;168:12706.

23. Fields JM, Lee PA, Jenq KY, et al. The interrater reliability of inferior vena cava ultrasound by bedside clinician sonographers in emergency department patients. Acad Emerg Med 2011;18:98-101.
24. Reinhart K, Kuhn HJ, Hartog C, Bredle DL. Continuous central venous and pulmonary artery oxygen saturation monitoring in the critically ill. Intens Care Med 2004;30:1572-8.

25. Thanakitcharu P, Charoenwut M, Siriwiwatanakul N. Inferior vena cava diameter and collapsibility index: a practical non-invasive evaluation of intravascular fluid volume in critically-ill patients. J Med Assoc Thailand 2013;96(Suppl.3):1422.

26. De Lorenzo RA, Morris MJ, Williams JB, et al. Does a simple bedside sonographic measurement of the inferior vena cava correlate to central venous pressure? J Emerg Med 2012;42:429-36.

27. Haydar SA, Moore ET, Higgins GL 3rd, et al. Effect of bedside ultrasonography on the certainty of physician clinical decision making for septic patients in the emergency department. Ann Emerg Med
2012;60:346-58.

28. Rivers EP, Ander DS, Powell D. Central venous oxygen saturation monitoring in the critically ill patient. Curr Opin Crit Care 2001;7:204-11.

29. Bakker J, Jansen TC. The prognostic value of blood lactate levels relative to that of vital signs in the pre-hospital setting. Crit Care 2008;12:R160.

30. Jones AE. Lactate clearance for assessing response to resuscitation in severe sepsis. Acad Emerg Med 2013;20:844-7.

31. Jones AE, Shapiro NI, Trzeciak S, et al. Lactate clearance vs central venous oxygen saturation as goals of early sepsis therapy: a randomized clinical trial. J Am Med Assoc 2010;303:739-46.

32. Jones AE. Point: should lactate clearance be substituted for central venous oxygen saturation as goals of early severe sepsis and septic shock therapy? Yes. Chest 2011;140:1406-8. 